\title{
STRAIN AND DAMAGE IN SILICON DUE TO A DEEP OXYGEN IMPLANTATION
}

THAD VREELAND, JR.* AND T.S. JAYADEV**

* Division of Engineering and Applied Science, California Institute of Technology, Pasadena, CA 91125

$\star \star$ Lockheed Research Laboratory, Palo Alto, CA 94304

\section{ABSTRACT}

Silicon wafers, implanted with oxygen at $550^{\circ} \mathrm{C}$ to form a buried oxide layer have been examined with TEM and x-ray diffraction. Implantation damage increased with depth up to $\sim 1500 \AA$ where some amorphous regions were seen. The amorphous region extended for $\sim 4500 \AA$ to a damaged crystalline region $\sim 1000 \AA$ thick. Double crystat x-ray rocking curves of the as-implanted and of annealed crystals were obtained and analyzed. The strain normal to the (100) surface in the as-implanted crystalline Si did not exceed $+0.05 \%$. After an argon anneal, the strain disappeared, leaving a crystal with a lower density of extended defects and no measureable misorientation of surface or deep layers. In marked contrast, an anneal in a hydrogen atmosphere exhibited no normal strain but contained regions above the oxide layer which differed in orientation from the substrate. The misorientation ranged up to $\sim 0.1$ degree.

\section{INTRODUCTION}

Several techniques have been pursued over the last few years to develop a viable silicon-on-insulator (SOI) technology. The technology has potential for the fabrication of radiation hard, dielectrically isolated, high speed integrated circuits. Recently, separation by implantation of oxygen (SIMOX) is showing signs of emerging as a practical SOI technology.

Stresses in SOI layers have posed serious problems in some SOI technologies, resulting in poor yields in processed circuits. It is well known that compressive stress is the cause of low carrier mobility in silicon-on-sapphire.

Hamdi, et al. investigated thermally annealed SIMOX samples (made by $\mathrm{O}_{2}{ }^{+}$ implantation) using RBS and ion channeling [1]. They found annealing in $\mathrm{Ar}$ produced slightly better top surface quality crystalline Si than annealing in $\mathrm{N}_{2}$. Wi lson and Fathy characterized SIMOX samples (made by $\mathrm{O}_{2}{ }^{+}$implantation) using RBS, TEM, and other analytical techniques,[2]. X-ray diffraction studies on as-implanted and annealed SIMOX crystals have not been reported. $X$-ray diffraction analysis is a sensitive tool for the study of crystalline solids. Double crystal diffractometry, where a nearly monochromatic plane wave is produced by diffraction from the highly perfect first crystal, is particularly suited to the study of the perfection of the second crystal. The analysis of double crystal x-ray rocking curves of single crystal structures can give valuable information on layer strains, displacement of atoms from normal lattice sites (which reduces the structure factor), crystallographic misorientations, and extended defects. Both strains and misorientations cause shifts in the Bragg angle. These two effects are readily separated using two rocking curves with appropriate hkl reflections. Both the thickness of a layer and the structure factor affect the integrated intensity of a Bragg peak while the width of the peak is affected by the thickness of the layer and its content of extended defects.

The double crystal rocking curve shows the reflecting power of the sample (diffracted intensity divided by the incident beam intensity) vs. deviation of the incident beam from the Bragg angle. The scattering of $x$-rays in thin crystal layers can be modeled to a very good approximation by the singlescattering or kinematical theory [3], while for thicker layers the dynamical theory must be used [4]. The rocking curve from a sample that has a uniform- 
ly strained layer on a relatively thick (approximately unstrained) substrate has a well defined zero order peak separated from the substrate Bragg peak. A series of smaller peaks of decreasing amplitude are formed on either side of the zero order peak when the crystal layers are of good quality. When the normal to the sample surface lies in the diffraction plane (defined by the incident and diffracted) beams, and $\phi$ is the rotation angle about the. surface normal, the angular separation of a misoriented layer is related to the strains [5] by

where

$$
\begin{aligned}
-\Delta \theta_{0} & =k_{1} \varepsilon^{\perp}+k_{2} \varepsilon^{\prime \prime} \pm \xi_{\phi} \\
k_{1} & =\cos ^{2} \psi \tan \theta_{B} \pm \sin \psi \cos \psi \\
k_{2} & =\sin ^{2} \psi \tan \theta_{B} \mp \sin \psi \cos \psi
\end{aligned}
$$

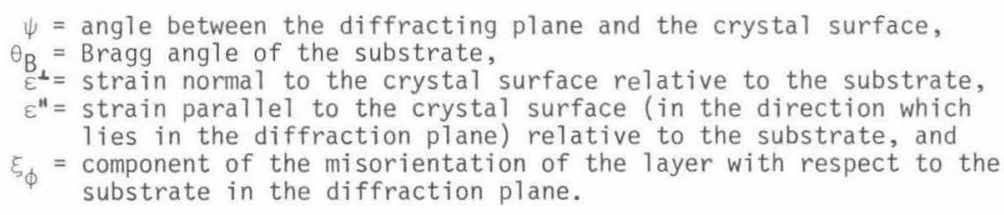

The upper sign in eqs. 1-3 is used when the angle of incidence of $x$-rays is $\theta_{B}-\psi$ from the surface, and the lower sign is used for $\theta_{B}+\psi$ incidence.

When the layer and substrate have the same free lattice parameter as in the present case, the strains determined from eq. 1 are the strains of elasticity theory, and they completely determine the strain tensor in a $\{100\}$ crystal layer with a surface free of tractions.

Two rocking curves, $a$ and $b$, taken with $\phi_{B}-\psi$ and $\theta_{B}+\psi$ incidence $\left(\phi=0^{\circ}\right.$ and $\phi=180^{\circ}$ ) respectively, give

and

$$
\begin{aligned}
& -\left(\Delta \theta_{0}{ }^{a}+\Delta \theta_{0}{ }^{b}\right) / 2=\left(\cos ^{2} \psi \varepsilon^{\perp}+\sin ^{2} \psi \varepsilon^{\prime \prime}\right) \tan \theta_{B} \\
& -\left(\Delta \theta_{0}^{a}-\Delta \theta_{0}^{b}\right) / 2=\xi_{\phi}+\left(\varepsilon^{\perp}-\varepsilon^{\prime \prime}\right) \sin \psi \cos \psi
\end{aligned}
$$

Equation 4 gives the peak shift with respect to the substrate due to the change in d spacing of the Bragg planes, while eq. 5 gives the rotation of the planes due to misorientation and strain. Two near-symmetric $\left(\psi \sim 0^{\circ}\right)$ rocking curves with $\phi=0$ and $180^{\circ}$ give $\varepsilon^{\perp}$ using eq. 4 , and two assymetric rocking curves then give $\varepsilon$ " using eq. 4 . The value of $\xi_{\phi}$ can then be found
using eq. 5 .

Speriosu, Paine, Nicolet, and Glass [6] investigated the strains in [100] Si due to Si implantation at ambient temperature. The strain perpendicular to the surface was found to increase linearly with dose up to a strain of about $1 \%$ at a dose of $\sim 10^{15} \mathrm{Si} / \mathrm{cm}^{2}$ where the structure became amorphous (230 keV implant and a $5200 \AA$ damaged layer).

Implanted silicon crystals with a buried oxide layer were examined with cross-sectional TEM in the present study. Rocking curves taken before and after annealing implanted crystals in argon and hydrogen atmospheres were obtained and analyzed.

\section{EXPERIMENTAL OBSERVATIONS AND ANALYSIS}

Silicon (100) wafers, p type $\sim 10 \mathrm{ohm}-\mathrm{cm}, 76 \mathrm{~mm}$, were implanted at $550^{\circ} \mathrm{C}$ and $160 \mathrm{keV}$ with a dose of $2.4 \times 10^{18} 0+/ \mathrm{cm}^{2}$. Wafer temperature was monitored by optical pyrometry during implantation. Following cleaning, wafers of implanted $\mathrm{Si}$ were annealed in hydrogen (io min at $1150^{\circ} \mathrm{C}$ in a horizontal epi reactor), and a $\sim 7000 \AA$ layer of boron doped Si was deposited by decomposition of $\mathrm{SiCl}_{4}$ at $1125^{\circ} \mathrm{C}$ in the reactor. Implanted wafers were also annealed for $2 \mathrm{~h}$ at $1100^{\circ} \mathrm{C}$ in flowing argon. Cooling rates $\sim 10^{\circ} \mathrm{C} / \mathrm{min}$ brought the wafers to ambient temperature. 

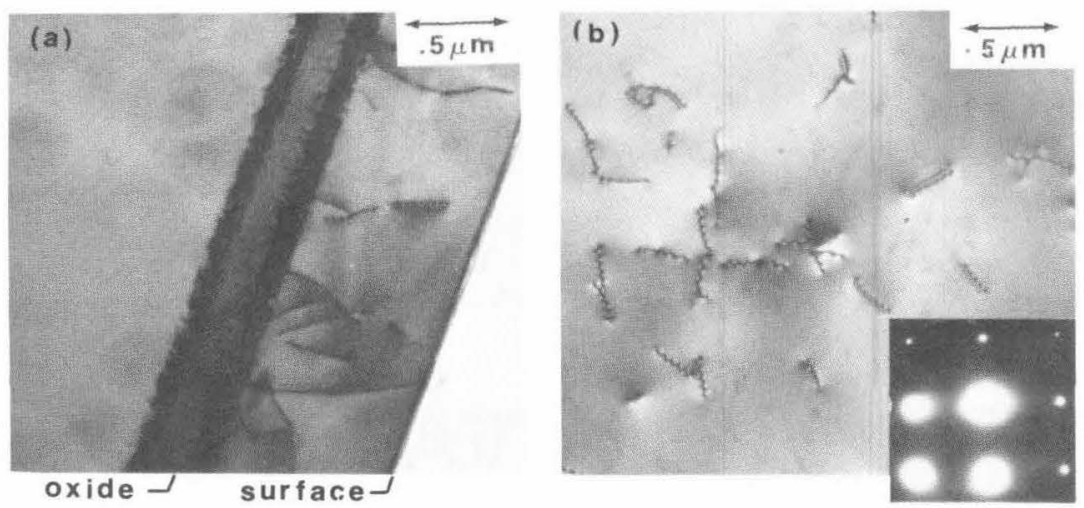

Fig. 1 (a) Cross-sectional transmission electron micrograph of the implanted and hydrogen annealed sample with a Si epilayer, (b) dislocations in the (100) surface region, density $\sim 10^{8} / \mathrm{cm}^{2}$.

A cross-sectional TEM micrograph of the hydrogen annealed sample with the Si epilayer is shown in Fig. 1(a). A $2000 \AA$ buried oxide layer is observed with $\sim 1000 \AA$ regions above and below it containing a high defect density. The crystal layer above the oxide is $\sim 8700 \AA$ thick (including the epilayer).

The normal to each crystal surface was found from a Laue back reflection $x$-ray pattern $\left(\psi \sim 2^{\circ}\right.$ for the (100) planes). X-ray rocking curves using $F e K \alpha_{1}$ radiation, with $\theta_{B}-\psi$ and $\theta_{B}+\psi x$-ray incidence were obtained on the as-implanted and annealed crystals. A GaAs (100) first crystal was used, and the (400) reflection from it served to select the $K \alpha_{1}$ and $K \alpha_{2} x$-ray lines. Slits placed between the GaAs crystal and the sample passed only the $\mathrm{K}_{1}$ line and limited the $x$-rays falling on the sample to an area of $0.5 \mathrm{~mm}^{2}$. A NaI(Tl) detector with pulse height analysis was used to measure $x$-ray intensities, and typical counting rates for the beam incident on the sample were $4 \times 10^{4}$ counts $/ \mathrm{s}$.

$X$-ray rocking curves of the as-implanted sample are shown in Fig. 2 .
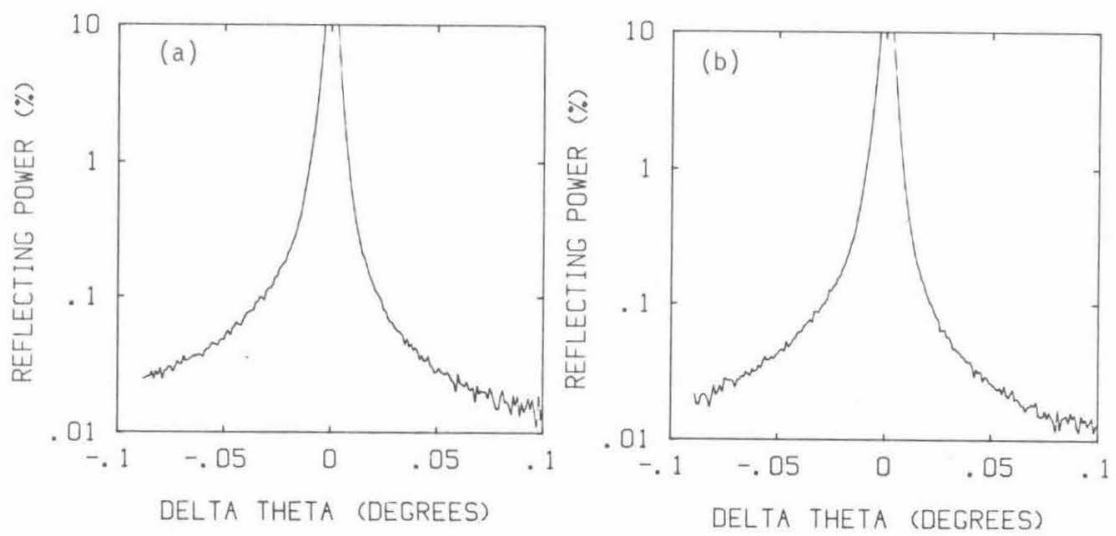

Fig. 2 (400) double crystal rocking curves of the as-implanted (100) Si, (a) $\theta_{B}+\psi$ incidence, (b) $\theta_{B}-\psi$ incidence, with $F e K \alpha_{1}$. 

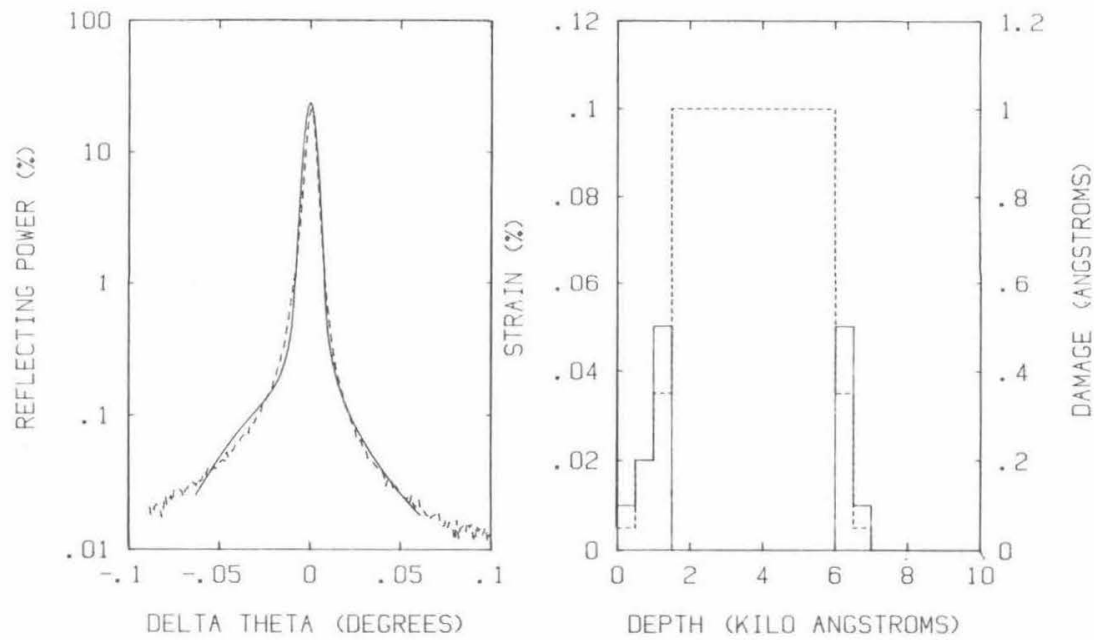

Fig. 3 (a) Calculated (solid) and experimental (dashed) (400) rocking curves of as-implanted $\mathrm{Si}\left(\theta_{\mathrm{B}}+\psi\right.$ incidence), (b) strain vs. depth (solid curve) and damage vs. depth (dashed curve) profiles used to calculate the rocking curve in (a).

The small asymmetry of the curves (slightly greater amplitude at incident angles less than the substrate Bragg angle (at $\Delta \theta=0^{\circ}$ ) indicate a small perpendicular strain in the Si. The parallel strain is not measured in this (nearly) symmetric rocking curve. (311) and (3TT) rocking curves with $\theta_{B}-\psi$ incidence show a similar asymmetry which indicates at most a small parallel strain ( $k_{1}=0.986$ and $k_{2}=-0.252$ for these reflections). The calculated $(400)$ rocking curve (kinematical with $\phi=180^{\circ}, k_{1}=0.982, k_{2}=0.036$ ) for the uniform layer strain and damage depth profiles in Fig. 3b are shown by the solid curve in Fig. 3a. The damage (dashed line in Fig. 3b) is the standard deviation of atoms from the normal lattice sites in angstroms (a damage of $\sim 1 \AA$ is equivalent to an amorphous state). The analysis assumed a uniform concentration of extended defects in the layers (the calculated rocking curve was convolved with a Gaussian of 45 arcsec standard deviation to account for the broadening due to extended defects), and the calculated curve when compared to the experimental (dashed) curve clearly indicates that strains larger than $\sim 0.05 \%$ are not present. Better agreement between experimental and calculated curves could be obtained with layers containing different densities of extended defects (as was observed in the TEM micrographs). The absence of large long range strains and the introduction of extended defects indicates that the sample was partially annealed during the $550^{\circ} \mathrm{C}$ implant.

Rocking curves of the implanted and hydrogen annealed $\mathrm{Si}$ are shown in Fig. $4 a\left(\phi=180^{\circ}\right)$. It is apparent that a shifted Bragg peak is present, and the shift is reversed in the two rocking curves. This indicates the absence of long range strain and the presence of misoriented crystal regions. The calculated curve (kinematical), assuming misoriented layers with thicknesses given in Table I, fits the experimental curve reasonably well as shown in Fig. 5 .

The total thickness of misoriented layers ( $8000 \AA$ in Table I) is equal to the thickness of the epilayer and the crystalline layer above the $1000 \AA^{\circ}$ layer with a high defect density. Oscillations in the rocking curve of the hydrogen annealed sample are absent, indicating a high density of extended defects in the misoriented layers (the calculated curve had to be convolved 


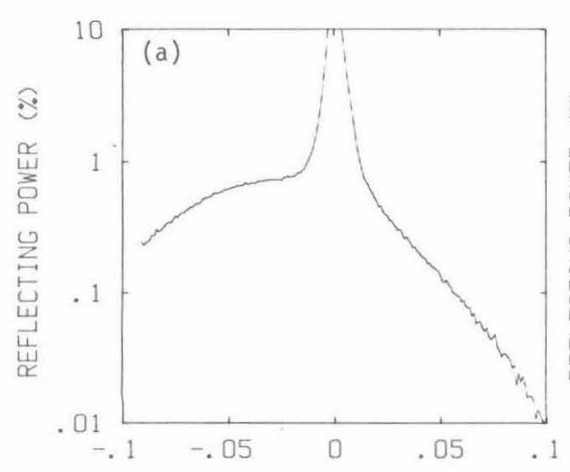

DELTA THETA (DEGREES)

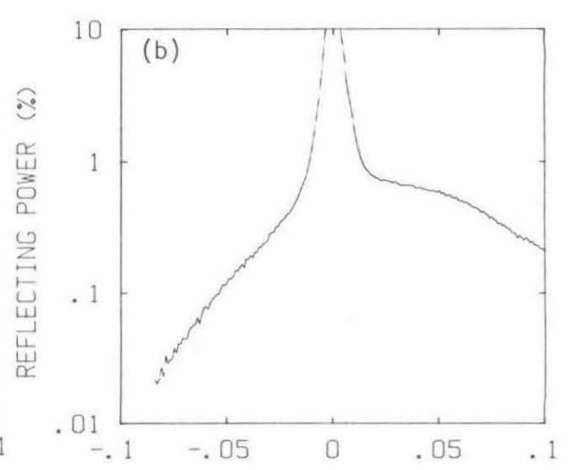

DELTA THETA (DEGREES)

Fig. 4 (400) double crystal rocking curves of implanted Si after a 10 min anneal in hydrogen at $1150^{\circ} \mathrm{C}$ and the growth of a Si epilayer.

(a) $\phi=0^{\circ}$, (b) $\phi=180^{\circ}$.

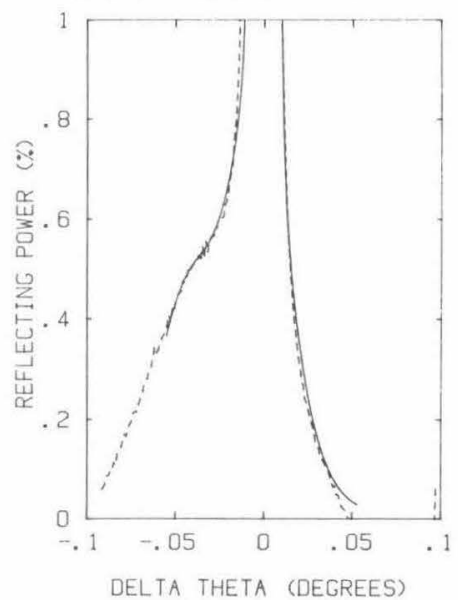

Fig. 5 Calculated (solid) and experimental (dashed) (400) rocking curves of implanted and hydrogen annealed Si.

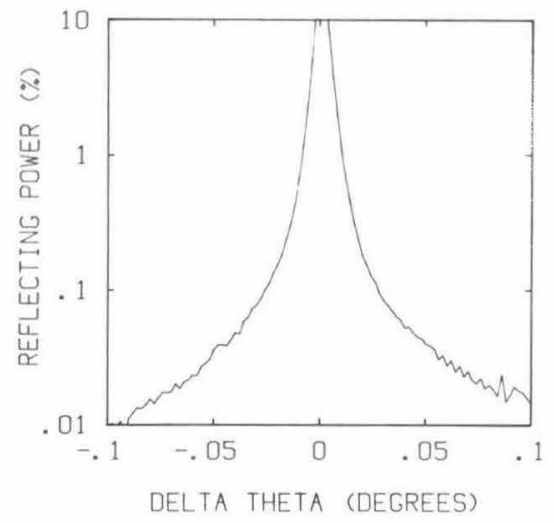

Fig. 6 (400) rocking curve of the implanted $\mathrm{Si}$ after a $2 \mathrm{~h}$ anneal in $\mathrm{Ar}$ at $1100^{\circ} \mathrm{C}$.

Table I ROTATION OF CRYSTAL LAYERS AND LAYER THICKNESS

$\begin{array}{cc}\text { ROTATION(degrees) } & \text { LAYER THICKNESS(angstroms) } \\ -0.011 & 1500 \\ 0 & 150 \\ 0.006 & 2000 \\ 0.014 & 1250 \\ 0.028 & 1150 \\ 0.056 & 1600 \\ 0.100 & 350\end{array}$


with a Gaussian of 55 arcsec standard deviation to eliminate the oscillations). The misorientations arise from dislocation arrays (subboundaries) which formed during the hydrogen anneal.

The formation of oxide causes a volume expansion, and if the process is not laterally uniform, dislocations will be generated and bending of the crystal overlayers will occur. An epilayer will replicate subboundaries in the crystal surface layer. Lateral non-uniformity in oxygen implanted and annealed samples has been observed in cross-sectional TEM micrographs which show oxide particles in the crystal layers adjacent to the buried oxide. The hydrogen anneal appears to produce more lateral non-uniformity than the argon anneal. Hydrogen-silicon bonds formed in the hydrogen anneal should reduce dislocation mobility and thereby hinder motion of dislocations to the Si surface. Oxide loss in the $\mathrm{H}_{2}$ anneal [7], if laterally non-uniform, could cause bending of the crystal overlayer.

The (400) $x$-ray rocking curve of the argon annealed sample is shown in Fig. 6. This curve very closely matches the curve obtained from a high quality Si (100) wafer, and indicates that the extended defect and point defect concentrations in the implanted layers were substantially reduced by the argon anneal.

\section{CONCLUSIONS}

1. The implantation of $2.4 \times 10^{18} / \mathrm{cm}^{2}, 180 \mathrm{keV}, 0^{+}$ions in $\mathrm{Si}$ results in a $2500 \AA$ crystalline surface layer with high dislocation density, on an amorphous layer $\sim 4500 \AA$ thick. A heavily damaged crystalline layer $\sim 1000 \AA$ thick is observed under the amorphous layer.

2. The long range strains in the crystalline layers above and below the amorphous layer are smal1 $(<0.05 \%)$.

3. Annealing in hydrogen $\left(10 \mathrm{~min}\right.$ at $1150^{\circ} \mathrm{C}$ ) relaxes the (sma11) long range strains and results in misoriented regions (up to $\sim 0.1^{\circ}$ ) in the surface crystalline layers and a buried oxide layer $\sim 1500 \AA$ thick.

4. Annealing in argon $\left(2 \mathrm{~h}\right.$ at $1100^{\circ} \mathrm{C}$ ) relaxes the (sma11) long range strains and results in a crystal with a buried oxide layer and no measureable misorientation in the surface crystalline layer.

\section{ACKNOWLEDGEMENTS}

This work was sponsored in part by the National Science Foundation under the Materials Research Group grant DMR 8421119 . The authors wish to thank Dr. P. Sioshansi of SPIRE for the oxygen implantation.

\section{REFERENCES}

1. A.H. Hamdi, F.D. McDaniel, R.F. Pinnizzotto, S. Matteson, H.W. Lam, and S.D.S. Malhi, Appl. Phys. Lett. 41, 1143 (1982).

2. S.R. Wilson and D. Fathy, J. Elect. Mat. 13, 127 (1984).

3. V.S. Speriosu, J. App1. Phys. 52, 6094 (1 $\overline{981})$.

4. C.M. Wei, T.A. Tombrelio, and T. Vreeland, Jr., submitted to J. Appl. Phys.

5. V.S. Speriosu and T. Vreeland, Jr., J. Appl. Phys. 56, 1591 (1984).

6. V.S. Speriosu, B.M. Paine, M-A. Nicolet and H. Glass, Appl. Phys. Lett. 40, 604 (1982).

7. K. Das, G. Shorthause, J. Butcher, and K.V. Anand, Microelectron. J. 14, $88(1983)$. 